\title{
Cultivation of paramecium caudatum in the presence of physiologically active substances, and a redox active polymer
}

\author{
Hiromasa Goto*, Kuniharu Nakajima \\ University of Tsukuba, Tsukuba, Ibaraki 305-8573, Japan \\ *E-mail address: gotoh@ims.tsukuba.ac.jp
}

\begin{abstract}
Cultivation of paramecium in the presence of physiologically active substances was carried out. We have monitored paramecium for 23 days in the solutions in the presence of physiologically active substances, and polyaniline as a redox active polymer.
\end{abstract}

Keywords: polyaniline; paramecium; physiologically active substance

\section{INTRODUCTION}

In this research, we carry out cultivation of paramecium caudatum in the presence of alginic acid, streptomycin, iota-carrageeenan, chondroitin sulfate sodium salt as physiologically active substances, and polyaniline as a redox active conducting polymer [1]. Polyaniline emeraldine base can be conveniently prepared by treatment of polyaniline emeraldine salt (as prepared polyaniline) with ammonia/water. Chemical structures of physiologically active substances employed in this study are indicated in Figure 1.

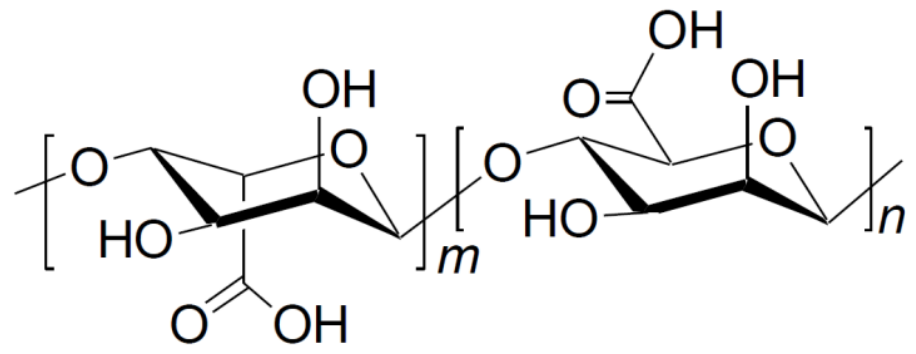

Alginic acid 


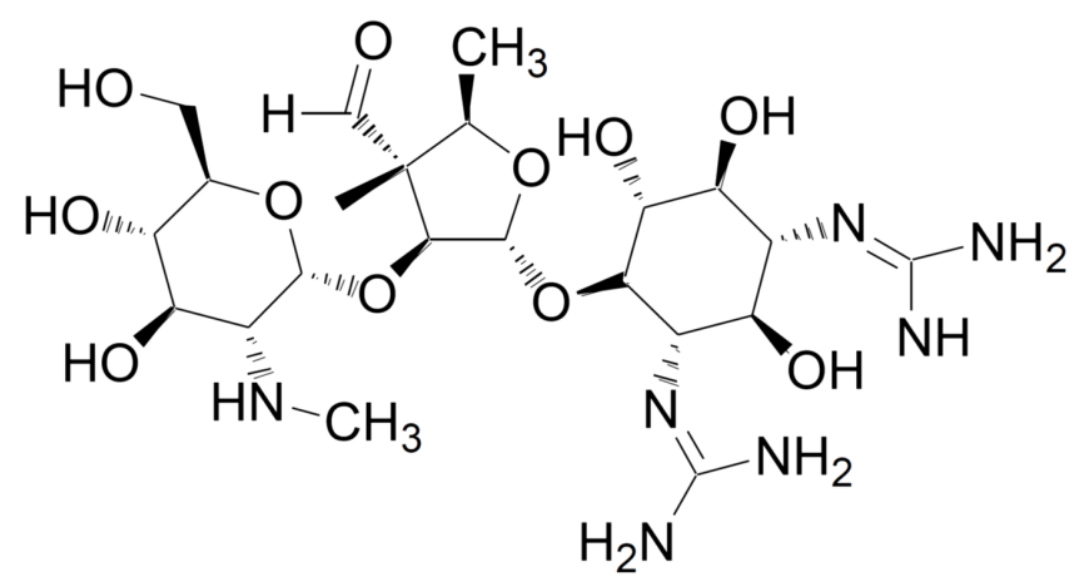

Streptomycin

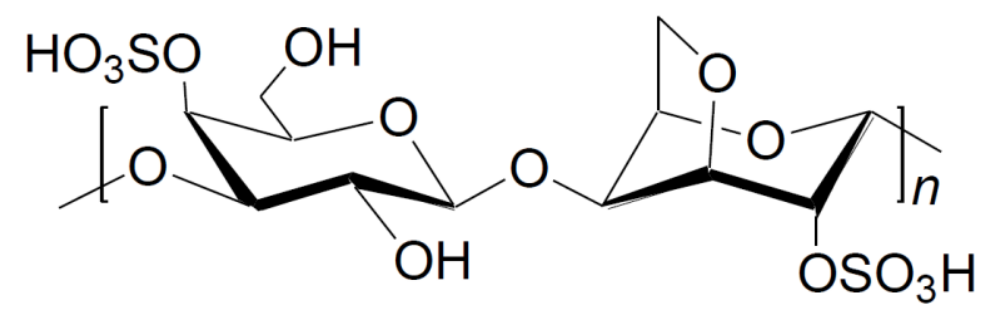

Iota-carrageeenan

Figure 1. Chemical structures of physiologically active substances employed in this study for cultivation of paramecium.

\section{EXPERIMENT}

\subsection{Synthesis of polyaniline [2]}

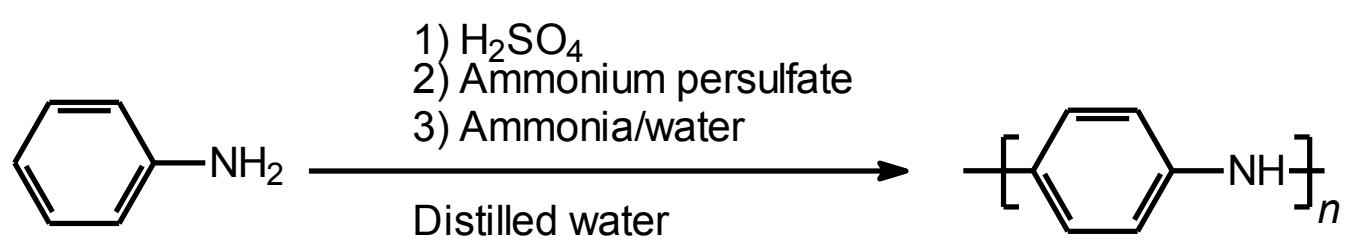

Scheme 1. Synthesis of polyaniline emeraldine base. 
A mixture of aniline $(1 \mathrm{~g})$, and sulfuric acid $(1 \mathrm{~g})$ in the distilled water $(200 \mathrm{~mL})$ was cooled with ice. Then, ammonium persulfate $(1.5 \mathrm{~g})$ was added to the solution and stirred for ca. $12 \mathrm{~h}$. The resultant green material was washed with large volume of water, and methanol. After filtration, the green cake on the filter was reduced in the ammonia/water. The colour of the polyaniline was changed from dark green to purple. The solution was filtered and dried in a vacuum to yield dark coloured powder as polyaniline emeraldine base (Scheme 1).

\subsection{Paramecium}

Paramecium caudatum was provided by Fujishima lab. (Yamaguchi Univ., Japan). Optical microscopy image of the paramecium is shown in Figure 2(a).

\subsection{Physiologically active substances}

Alginic acid, streptomycin, iota-carrageeenan, and chondroitin sulfate sodium salt were purchased from Tokyo Chemical Industry (TCI). The reagents were used as received.

\subsection{Cultivation}

Cultivation of paramecium was carried out in a solution containing paramecium $(5 \mathrm{~mL})$, $1 \mathrm{mg}$ of physiologically active substance (alginic acid, streptomycin, iota-carrageeenan, or chondroitin sulfate sodium salt), or polyaniline $(5 \mathrm{~mL})$ in $5 \mathrm{~mL}$ of distilled water, as shown in Figure 2.

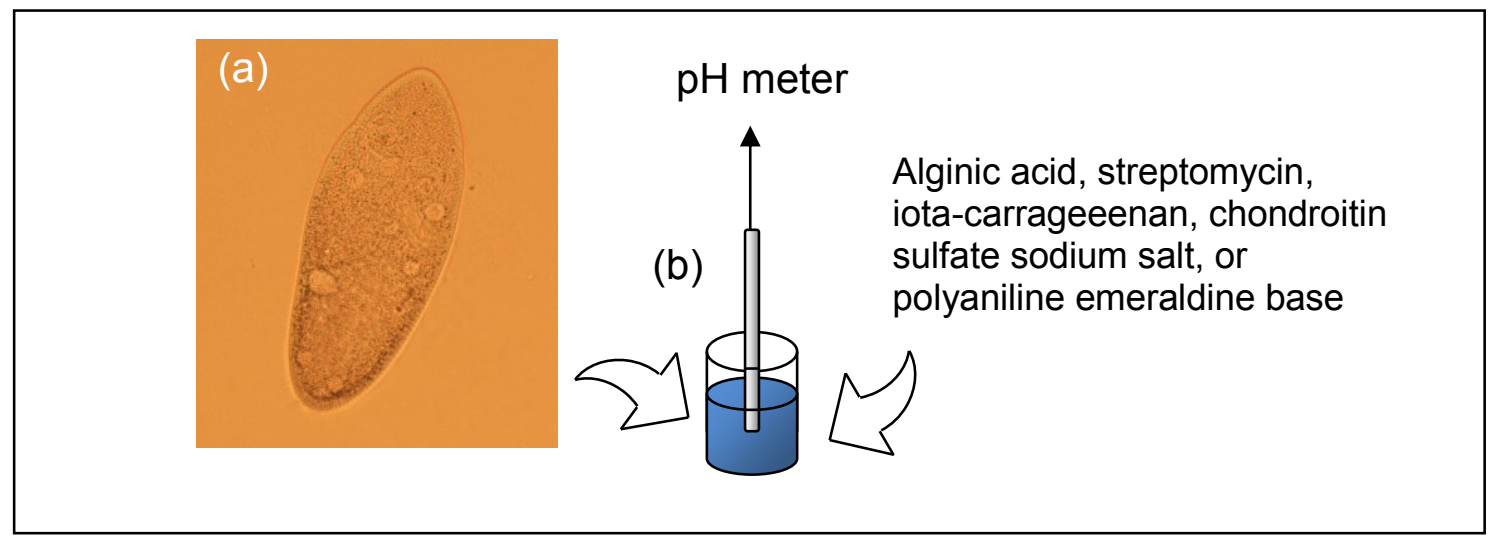

Figure 2. Cultication of paramecium. (a): Optical microscopy image of paramecium.

(b): cultivation vessel.

\section{RESULTS AND DISCUSSION}

The paramecium lived for 17 days in the water solution in the presence of alginic acid, and streptomycin. Furhermore, the paramecium lived for 23 days in the solution in the presence of chondroitin sulfate sodium salt, and polyaniline. Waste (ammonia) from paramecia results in poison for living environment. Polyaniline emeraldine base can absorb the discharged waste through neutralization reaction, improving water environment for life. The $\mathrm{pH}$ value of the water in the presence of polyaniline was somewhat increased from 7.48 to 8.38 in this experiment after 23 days. Precise estimation of appropreate amount of 
polyaniline and physiologically active substances for paramecium, and consideration of chemical interaction between the substances and paramecium are required for effective cultivation.

\section{Acknowledgement}

Paramecium strain (P. caudatum KNZ1207 (PC012005A)) used in this study was provided by Symbiosis Laboratory, Yamaguchi University with support in part by the National Bio-Resource Project of the Ministry of Education, Culture, Sports, Science and Technology, Japan.

\section{Reference}

[1] H. Goto, IOP Conf. Ser.: Mater. Sci. Eng. 54 (2014) 012024.

[2] A. G. MacDiarmid, J.-C. Chiang, M. Halpern, W.-S. Huang, S.-L. Mu, L. D. Nanaxakkara, S. W. Wu, S. I. Yaniger, Mol. Cryst. Liq. Cryst. 121 (1985) 173-180. 